\title{
The Effect of Payout Ratio Dividend and Earning Volatility on Stock Price (In the 2014-2017 BEI Manufacturing Company)
}

\author{
Jajang Badruzaman
}

Accounting Study Program, Faculty of Economics and Business, Siliwangi University, J1 Siliwangi No.24, Kahuripan, Kec Tawang, Tasikmalaya, Jawa Barat 46115, Indonesia

DOI: $10.36348 /$ sjef.2020.v04i07.004 | Received: 02.07.2020 | Accepted: 09.07.2020 | Published: 16.07 .2020

*Corresponding author: Jajang Badruzaman

\section{Abstract}

This research entitled The Effect of Dividend Payout Ratio and Earning Volatility on Stock Prices, the aim is to find out the effect of dividend payout ratio and earnings volatility on stock prices on manufacturing companies in the Indonesia Stock Exchange in 2014-2017 both in parsila maupu together. The sampling technique used purposive sampling and data analysis used panel data regression. The results of data processing show that the Dividend Payout Ratio has no significant effect on the Stock Price. Then Earning Volatility has a significant effect on Stock Prices and jointly with Payout Ratio and Earning Volatility has a significant effect on Stock Prices.

Keywords: Dividen Payout Ratio, Earning Volatility, Harga Saham.

Copyright @ 2020: This is an open-access article distributed under the terms of the Creative Commons Attribution license which permits unrestricted use, distribution, and reproduction in any medium for non-commercial use (NonCommercial, or CC-BY-NC) provided the original author and source are credited.

\section{INTRODUCTION}

The capital market is a place that can be used by businesses and investors to buy and sell various long-term financial instruments including stocks, bonds and derivative instruments. The capital market plays a role in supporting the success of meeting the funding needs of the business world, as well as investors as those who have excess funds can invest their funds in various securities in the hope of getting a return on that investment.

One form of long-term financial instruments that are traded on the capital market and much sought after by the public is stocks. Stocks have the ability to generate higher profits compared to other capital market instruments, so in investing an investor needs to first analyze the company that is his investment destination.

Investors need a variety of information, both fundamental and technical information. Fundamental information is information that is produced by companies such as financial reports. While technical information is information generated outside the company but will have an impact on the company, for example information about stock price movements and stock price indexes. Both of these information investors must do their analysis so that investors in making decisions will be right, both in buying or selling shares.
Based on the explanation above, the writer is interested in conducting a study entitled The Effect of Dividend Payout Ratio and Earning Volatility on Stock Prices in Manufacturing Companies Listed on the Indonesia Stock Exchange in 2014-2017.

\section{LITERATURE REVIEW}

Dividends are the portion of profits distributed to shareholders [1]. Meanwhile, according to Bambang Riyanto [2], "Dividends are cash flow paid to shareholders or equity investors". Distribution of ordinary stock dividends can be done if the company has paid a preferred stock dividend [3].

Brigham et al., [4] there are five types of dividends as follows:
a. Cash Dividend (cash dividend)
b. Stock Dividend (stock dividend)
c. Property Dividend
d. Dividend Scrip
e. Liquidating dividend

Dividend policy is an integral part of a company's funding decision. The dividend payout ratio determines the amount of profit that can be retained as a source of funding. The greater the retained earnings the less the amount of profit allocated for dividend payments. 
The definition of dividend payout ratio according to Sartono [5] states that "The dividend payout ratio is the percentage of profit paid in the form of dividends with the total profit available to shareholders".
Meanwhile, according to Sutrisno [6] "Dividend Payout Ratio is the percentage of profit distributed as dividends, where the greater the Dividend Payout Ratio the smaller the portion of funds available to be reinvested to the company as retained earnings".

Mathematically Dividend Payout Ratio can be formulated as follows:

$$
\text { Dividend Payout Ratio }=\frac{\text { Dividend Per Share }}{\text { Earning Per Share }} \times 100 \%
$$

Tjiptono Darmaji, 2006 [7]

Then Van Horne, James C., John M. Wachowicz, Jr. [8] there are several factors that influence dividend policy, which are as follows:

1. Company liquidity

2. The need for funds to pay debts

3 . The company's growth rate

4. Opportunities to the capital market

5. Supervision of fund companies originating

Candra Puspita Ningtyas, Suhadak and Nila Firdausi Nuzula [9] that the Industry Standard Dividend Payout Ratio (DPR) is 5.21\%. So Dividend Payout Ratio (DPR) which is above the industry standard is a company that is classified as good or healthy while Dividend Payout Ratio (DPR) which is below the industry average is a company that is classified as not good or unhealthy.

\section{Earning Volatility}

Earnings volatility is a statistical concept that determines the associated risk and helps predict the market price of a particular stock financial term [10]. While volatility is a measure that states how much the level of fluctuations (price changes) on certain assets [11].

According to Khurniaji [12] earnings volatility which is a measure that illustrates the extent of the level of stability of earnings or revenue generated by the company from year to year. Theresia and Arilyn [13] states that earnings volatility is the profit fluctuation of a company that reflects the risk of the company's operating activities, this will affect investors' decisions regarding the risks that occur in a company.

Subiyakti [14] earnings volatility is the movement of income changes. If a volatility in the company is high, then the level of profit or income tendencies in the company can be lost easily. And if the volatility of income from a small company, then the company tends to be constant and has no change in production.

Based on some of the above understanding, it can be concluded that earnings volatility is a measure that reflects the ups and downs of profits derived by the company within a certain period that reflects the risk of the company's operating activities. Earnings Volatility will have an impact on 1. Cost of capital, 2. Earnings predictability and 3. Share prices [10].

Earning volatility or income volatility illustrates the level of business risk and the level of bankruptcy of the company, the increase in earnings volatility will increase the risk of loss of company profits, and if it occurs continuously will disrupt the operational activities of the company [12]. Referring to research conducted by Sinta Adelia Lubis [15], measurement of earnings volatility uses a comparison between operating profit and total assets.

Earninig Volatility $=($ Operating Profit $) /($ Total Assets)

\section{Stock Prices}

Brigham, Eugene F., Joel F. Houston [4] stock prices determine shareholder wealth. Maximizing shareholder wealth translates into maximizing the company's stock price. The price of a stock at a certain time will depend on the cash flow that is expected to be received in the future by the "average" investor if the investor buys the stock.

According to Takarini and Hendrarini [16] states that the stock price is one indicator of a company in achieving success.

According to Darmaji and Fakhrudin [17] stock prices are prices that occur on the stock exchange at a certain time. Stock prices can change up or down in a matter of time that is so fast, can change in minutes can even change in seconds. This is possible because it depends on the demand and supply between the buyer of shares and the seller of shares."

Hadi [18] stated that the stock price is the value of shares in rupiah formed by the action of buying and offering shares on the stock exchange by fellow exchange members. Meanwhile, according to Zulfikar [19] said that stock prices that occur in the capital market always fluctuate from time to time. The price fluctuation will be determined by the strength of supply and demand. If the number of offers is greater than the number of requests, in general the price exchange rate drops. Conversely, if the number of requests is greater than the number of bids, the stock price tends to rise. 
Based on the above understanding it can be concluded that the stock price is the price that occurs in the capital market at a certain time that is formed according to market demand and supply and is usually a closing price. In a piece of stock paper has a value or price. According to Widoatmojo [20] the types of stock prices are as follows:
1. Nominal Prices
2. Prime Price
3. Market Prices
4. Opening Price
5. Closing Price
6. Highest Price
7. Lowest price
8. Average Prices

There are several factors that affect the price of securities [21]:

1. Earnings-related announcements.

2. Forecasting announcements by company officials (forecast announcements by company official).

3. Dividend announcements.

4. Financing announcements (financing announcements).

5. Announcements relating to the government.

6. Investment announcements.

7. Labor announcements.

8. Legal announcements (legal announcements).

9. Marketing-production-sales announcements.

10. Management announcements (managementboard of director announcements),

11. Announcements of merger take-divestiture announcements.

12. Securities industry announcements (securities industry announcements).

\section{RESEARCH METHODS}

The research method used by the author in this research is descriptive method. According to Sugiyono [22], the research method is a scientific way to obtain data with specific goals and uses, the scientific way means the research activities are based on scientific characteristics, namely rational, empirical and systematic.

\section{Operationalization of Variables}

According to Sugiyono [23], variables are anything in the form of what is determined by researchers to be studied in order to obtain information about it, on this occasion the variables studied included the Independent Variable (Dividend Pay Out Ratio (X1) and Earning Volatility (X2) and Dependent Variable is the Share Price $(\mathrm{Y})$.

\section{Data Collection Technique}

In this study, the authors used secondary data collection techniques. Secondary data is a source that does not directly provide data to data collectors [22].

\section{Target Population}

The target population in this study are all manufacturing companies listed on the Indonesia Stock Exchange as many as 128 industrial companies. Based on the variables examined from 2014 to 2017, there were 25 companies that could be studied.

\section{Data Collection Procedure}

The procedure used by the authors in this research is library research. Literature study is research that studies the literature of books and other media relating to the problem to be examined. Data obtained from the official website of the Indonesia Stock Exchange. Yahoo Finance, id investing, as well as books, journals and other written works that support and are considered able to provide input in this research.

\section{Research Model}

The research model is a thinking pattern that shows the relationship between variables to be studied which also reflects the formulation of the problem that needs to be answered through research [24].

According to Sugiyono [22], data analysis is the process of systematically searching and compiling data obtained from interviews, field notes and other materials, so that they can be easily understood, and their findings can be shared with others. Data analysis techniques in this study using panel data (pooled data) so that the regression using panel data is called the panel data regression model.

The panel data regression model is as follows:

$$
Y=\alpha+\beta_{1} X_{1} \text { it }+\beta_{2} X_{2} \text { it }+\beta_{3} X_{3} \text { it }+\beta_{4} X_{4} \text { it }+e
$$

Information:

$$
\begin{array}{ll}
\mathrm{Y} & =\text { Stock Price } \\
\mathrm{a} & =\text { constant } \\
\beta_{1,2,3,4}= & \text { Regression Coefficient } \\
\mathrm{X}_{1} & =\text { Dividen Pau Out Ratio } \\
\mathrm{X}_{2} & =\text { Earning Volatility } \\
\mathrm{t} & =\text { time } \\
\mathrm{i} & =\text { company } \\
\mathrm{e} & =\text { Error term }
\end{array}
$$

\section{Classical Panel Data Assumptions Test}

The classic assumption test is a test conducted to see whether in a research model there are problems that will interfere with the results of the significance of the study. According to Agus Tri Basuki and Nano Prawoto [25] the classic assumption test in linear regression with the Ordinary Least Square (OLS) approach covers the tests of linearity, autocorrelation, heteroscedasticity, multicollinearity and normality

\section{Panel Data Regression Estimation Technique}

According to Basuki \& Prawoto [25], to estimate model parameters with panel data, there are 3 (three) techniques used, namely Common Effect Model, 
Fixed Effect Model and Random Effect Model. As for testing the model carried out with 3 tests, namely the Chow test, the Hausman test and the Lagrange multiplier test Basuki \& Prawoto [25].

a. Hypothesis Testing

a. $\mathrm{T}$ test (Partial Test)

$$
t=\frac{r \sqrt{n-2}}{\sqrt{1-r^{2}}}
$$

Information:

$\mathrm{t}=$ Distribution $\mathrm{t}$

$r=$ Partial correlation coefficient

$r^{2}=$ coefficient of determination

$\mathrm{n}=$ Amount of data b. Collaborative Testing (F-Test)

$$
\mathrm{F}=\frac{\mathrm{R}^{2} \mathrm{~K}}{\left(1-\mathrm{R}^{2}\right) /(\mathrm{n}-\mathrm{k}-1)}
$$

Sugiyono [22]

Information:

$$
\mathrm{R}^{2}=\text { coefficient of determination }
$$

Koefisien determinasi

$$
\begin{array}{ll}
\mathrm{K} & =\text { Number of independent variables } \\
\mathrm{n} & =\text { Number of data members or cases }
\end{array}
$$

c. Coefficient of Determination

$$
\mathrm{K}_{\mathrm{d}}=\mathrm{R}^{2} \times 100 \%
$$

Information:

$\mathrm{K}_{\mathrm{d}} \quad$ : The coefficient of determination

$\mathrm{R}^{2} \quad$ : The correlation coefficient is squared

\section{Attachment}

Table-1: Dividen Payout Ratio (DPR) Industry Manufacture Period 2014-2017

\begin{tabular}{|l|l|l|l|l|l|l|}
\hline No & CODE & Company & \multicolumn{4}{l}{ Deviden Payout Ratio } \\
\cline { 4 - 7 } & & & $\mathbf{2 0 1 4}$ & $\mathbf{2 0 1 5}$ & $\mathbf{2 0 1 6}$ & $\mathbf{2 0 1 7}$ \\
\hline 1 & ASII & Astra International Tbk. & 45,59 & 49,54 & 44,87 & 39,67 \\
\hline 2 & AUTO & Astra Otoparts Tbk. & 53,08 & 40,85 & 10,37 & 28,84 \\
\hline 3 & BATA & Sepatu Bata Tbk. & 40 & 6,47 & 73,17 & 51,46 \\
\hline 4 & BRAM & Indo Kordsa Tbk. & 26,23 & 36,86 & 26,08 & 59,51 \\
\hline 5 & CINT & Chitose Internasional Tbk. & 24,4 & 28,11 & 25,9 & 28,92 \\
\hline 6 & CPIN & Charoen Pokphand Indonesia Tbk & 16,9 & 25,89 & 413,54 & 36,76 \\
\hline 7 & EKAD & Ekadharma International Tbk. & 15,71 & 14,82 & 25,46 & 16,69 \\
\hline 8 & GGRM & Gudang Garam Tbk. & 28,67 & 77,73 & 74,92 & 64,51 \\
\hline 9 & HMSP & H.M. Sampoerna Tbk. & 86,45 & 99,89 & 98,16 & 98,5 \\
\hline 10 & ICBP & Indofood CBP Sukses Makmur Tbk & 49,71 & 49,75 & 24,94 & 49,76 \\
\hline 11 & IMAS & Indomobil Sukses Internasional & $-21,57$ & $-60,47$ & $-4,78$ & $-12,61$ \\
\hline 12 & INDF & Indofood Sukses Makmur Tbk. & 49,72 & 49,7 & 49,79 & 49,92 \\
\hline 13 & INKP & Indah Kiat Pulp \& Paper Tbk. & 12,21 & 4,19 & 6,03 & 9,77 \\
\hline 14 & INTP & Indocement Tunggal Prakarsa Tb & 94,29 & 35,07 & 88,36 & 138,55 \\
\hline 15 & KLBF & Kalbe Farma Tbk. & 43,11 & 44,44 & 44,84 & 48,75 \\
\hline 16 & MLBI & Multi Bintang Indonesia Tbk. & 0,68 & 145,92 & 100 & 99,95 \\
\hline 17 & SIDO & Industri Jamu dan Farmasi Sido & 86,71 & 85,72 & 81,16 & 81,49 \\
\hline 18 & SMBR & Semen Baturaja (Persero) Tbk. & 25 & 25 & 25 & 25 \\
\hline 19 & SMSM & Selamat Sempurna Tbk. & 42,7 & 62,28 & 20,66 & 71,49 \\
\hline 20 & SRIL & Sri Rejeki Isman Tbk. & 12,97 & 6,84 & 6,99 & 13,63 \\
\hline 21 & TKIM & Pabrik Kertas Tjiwi Kimia Tbk. & 10,49 & 62,76 & 174,53 & 25,24 \\
\hline 22 & TOTO & Surya Toto Indonesia Tbk. & 28,66 & 43,42 & 79,59 & 48,1 \\
\hline 23 & TRIS & Trisula International Tbk. & 40,59 & 37,13 & 79,14 & 350,9 \\
\hline 24 & UNVR & Unilever Indonesia Tbk. & 44,67 & 99,88 & 99,69 & 99,67 \\
\hline 25 & WTON & Wijaya Karya Beton Tbk. & 31,36 & 31,38 & 31,35 & 31,36 \\
\hline
\end{tabular}


Table-2: Earning Volatility Industry Manufacture Period 2014-2017

\begin{tabular}{|l|l|l|l|l|l|l|}
\hline No & CODE & Company & \multicolumn{3}{l|}{ Earning Volatility } \\
\cline { 4 - 7 } & & & $\mathbf{2 0 1 4}$ & $\mathbf{2 0 1 5}$ & $\mathbf{2 0 1 6}$ & $\mathbf{2 0 1 7}$ \\
\hline 1 & ASII & Astra International Tbk. & 11,59 & 8 & 8,5 & 9,88 \\
\hline 2 & AUTO & Astra Otoparts Tbk. & 7,71 & 3,02 & 4,44 & 4,82 \\
\hline 3 & BATA & Sepatu Bata Tbk. & 12,77 & 18,71 & 8,11 & 9,29 \\
\hline 4 & BRAM & Indo Kordsa Tbk. & 7,07 & 6,27 & 10,48 & 11,19 \\
\hline 5 & CINT & Chitose Internasional Tbk. & 9,82 & 10,65 & 7,05 & 8,04 \\
\hline 6 & CPIN & Charoen Pokphand Indonesia Tbk & 10,1 & 9,24 & 16,46 & 13,28 \\
\hline 7 & EKAD & Ekadharma International Tbk. & 14,28 & 17,02 & 16,86 & 12,88 \\
\hline 8 & GGRM & Gudang Garam Tbk. & 12,38 & 13,6 & 14,19 & 15,63 \\
\hline 9 & HMSP & H.M. Sampoerna Tbk. & 48,34 & 36,65 & 40,02 & 39,16 \\
\hline 10 & ICBP & Indofood CBP Sukses Makmur Tbk & 13,6 & 15,1 & 17,26 & 16,47 \\
\hline 11 & IMAS & Indomobil Sukses Internasional & 0,08 & 8,1 & $-0,88$ & 0,47 \\
\hline 12 & INDF & Indofood Sukses Makmur Tbk. & 7,25 & 5,4 & 8,99 & 8,71 \\
\hline 13 & INKP & Indah Kiat Pulp \& Paper Tbk. & 1,94 & 3,27 & 2,11 & 5,99 \\
\hline 14 & INTP & Indocement Tunggal Prakarsa Tb & 2,82 & 2,13 & 1,66 & 1,43 \\
\hline 15 & KLBF & Kalbe Farma Tbk. & 22,23 & 19,87 & 20,3 & 19,51 \\
\hline 16 & MLBI & Multi Bintang Indonesia Tbk. & 48,33 & 32,16 & 58,03 & 70,91 \\
\hline 17 & SIDO & Industri Jamu dan Farmasi Sido & 19,34 & 20,04 & 21,06 & 21,59 \\
\hline 18 & SMBR & Semen Baturaja (Persero) Tbk. & 13,49 & 13,57 & 7,99 & 39,9 \\
\hline 19 & SMSM & Selamat Sempurna Tbk. & 30,93 & 26,29 & 291,92 & 29,49 \\
\hline 20 & SRIL & Sri Rejeki Isman Tbk. & 9,44 & 8,24 & 6,97 & 7,1 \\
\hline 21 & TKIM & Pabrik Kertas Tjiwi Kimia Tbk. & 0,41 & $-0,43$ & 0,04 & 1,38 \\
\hline 22 & TOTO & Surya Toto Indonesia Tbk. & 18,84 & 15,64 & 9,74 & 13,36 \\
\hline 23 & TRIS & Trisula International Tbk. & 9,1 & 8,73 & 7,5 & 4,01 \\
\hline 24 & UNVR & Unilever Indonesia Tbk. & 53,76 & 49,77 & 51,19 & 49,57 \\
\hline 25 & WTON & Wijaya Karya Beton Tbk. & 10,82 & 4,62 & 7,3 & 5,94 \\
\hline
\end{tabular}

www.idx.co.id

Table-3: Stock Price Industry Manufacture Period 2014-2017

\begin{tabular}{|l|l|l|l|l|l|l|}
\hline No & \multirow{2}{*}{ KODE } & Nama Perusahaan & \multicolumn{4}{l}{ Harga Saham $($ Closing Price $)$} \\
\cline { 4 - 7 } & & & $\mathbf{2 0 1 4}$ & $\mathbf{2 0 1 5}$ & $\mathbf{2 0 1 6}$ & $\mathbf{2 0 1 7}$ \\
\hline 1 & ASII & Astra International Tbk. & 7.425 & 6.000 & 8.275 & 8.300 \\
\hline 2 & AUTO & Astra Otoparts Tbk. & 4.200 & 1.600 & 2.050 & 2.060 \\
\hline 3 & BATA & Sepatu Bata Tbk. & 1.105 & 900 & 790 & 570 \\
\hline 4 & BRAM & Indo Kordsa Tbk. & 5.000 & 4.680 & 6.675 & 7.375 \\
\hline 5 & CINT & Chitose Internasional Tbk. & 362 & 338 & 316 & 334 \\
\hline 6 & CPIN & Charoen Pokphand Indonesia Tbk & 3.780 & 2.600 & 3.090 & 3.000 \\
\hline 7 & EKAD & Ekadharma International Tbk. & 515 & 400 & 590 & 695 \\
\hline 8 & GGRM & Gudang Garam Tbk. & 60.700 & 55.000 & 63.900 & 83.800 \\
\hline 9 & HMSP & H.M. Sampoerna Tbk. & 68.650 & 94.000 & 3.830 & 4.730 \\
\hline 10 & ICBP & Indofood CBP Sukses Makmur Tbk & 13.100 & 13.475 & 8.575 & 8.900 \\
\hline 11 & IMAS & Indomobil Sukses Internasional & 4.000 & 2.365 & 1.310 & 840 \\
\hline 12 & INDF & Indofood Sukses Makmur Tbk. & 6.750 & 5.175 & 7.925 & 7.625 \\
\hline 13 & INKP & Indah Kiat Pulp \& Paper Tbk. & 1.045 & 955 & 955 & 5.400 \\
\hline 14 & INTP & Indocement Tunggal Prakarsa Tb & 25.000 & 22.325 & 5.400 & 1.950 \\
\hline 15 & KLBF & Kalbe Farma Tbk. & 1.830 & 1.320 & 1.515 & 1.690 \\
\hline 16 & MLBI & Multi Bintang Indonesia Tbk. & 12.100 & 8.200 & 1.750 & 13.675 \\
\hline 17 & SIDO & Industri Jamu dan Farmasi Sido & 610 & 550 & 520 & 545 \\
\hline 18 & SMBR & Semen Baturaja (Persero) Tbk. & 381 & 291 & 2.790 & 3.800 \\
\hline 19 & SMSM & Selamat Sempurna Tbk. & 4.750 & 4.760 & 980 & 1.255 \\
\hline 20 & SRIL & Sri Rejeki Isman Tbk. & 164 & 389 & 230 & 350 \\
\hline 21 & TKIM & Pabrik Kertas Tjiwi Kimia Tbk. & 850 & 495 & 730 & 2.920 \\
\hline 22 & TOTO & Surya Toto Indonesia Tbk. & 3.975 & 6.950 & 498 & 408 \\
\hline 23 & TRIS & Trisula International Tbk. & 388 & 300 & 336 & 308 \\
\hline 24 & UNVR & Unilever Indonesia Tbk. & 32.300 & 37.000 & 8.800 & 55.900 \\
\hline 25 & WTON & Wijaya Karya Beton Tbk. & 1.300 & 825 & 825 & 825 \\
\hline
\end{tabular}


Multicollinearity Test

\begin{tabular}{|l|l|l|}
\hline & DPR & EVOL \\
\hline DPR & 1.000000 & 0.023599 \\
\hline EVOL & 0.023599 & 1.000000 \\
\hline
\end{tabular}

Heteroscedasticity Test

\begin{tabular}{|c|c|c|c|c|}
\hline \multicolumn{5}{|c|}{ Dependent Variable: RESABS } \\
\hline \multicolumn{5}{|c|}{ Method: Panel Least Squares } \\
\hline \multicolumn{5}{|c|}{ Date: $06 / 28 / 20$ Time: $18: 40$} \\
\hline \multicolumn{5}{|c|}{ Sample: 20142017} \\
\hline \multicolumn{5}{|c|}{ Periods included: 4} \\
\hline \multicolumn{5}{|c|}{ Cross-sections included: 24} \\
\hline \multicolumn{5}{|c|}{ Total panel (balanced) observations: 96} \\
\hline Variable & Coefficient & Std. Error & $\mathrm{t}$-Statistic & Prob. \\
\hline $\mathrm{C}$ & 90.00670 & 14.54831 & 6.186746 & 0.0000 \\
\hline DPR & -0.055596 & 0.171016 & -0.325089 & 0.7461 \\
\hline EVOL & 0.232567 & 0.350312 & 0.663885 & 0.5089 \\
\hline \multicolumn{5}{|c|}{ Effects Specification } \\
\hline \multicolumn{5}{|c|}{ Cross-section fixed (dummy variables) } \\
\hline R-squared & 0.773692 & \multicolumn{2}{|c|}{ Mean dependent var } & 91.27498 \\
\hline Adjusted R-squared & 0.692868 & \multirow{2}{*}{\multicolumn{2}{|c|}{$\begin{array}{l}\text { S.D. dependent var } \\
\text { Akaike info criterion }\end{array}$}} & 146.0423 \\
\hline S.E. of regression & 80.93584 & & & 11.85100 \\
\hline Sum squared resid & 458542.7 & \multicolumn{2}{|c|}{ Schwarz criterion } & 12.54552 \\
\hline Log likelihood & -542.8482 & \multicolumn{2}{|c|}{ Hannan-Quinn criter. } & 12.13174 \\
\hline F-statistic & 9.572542 & \multicolumn{2}{|c|}{ Durbin-Watson stat } & 1.790049 \\
\hline Prob(F-statistic) & 0.000000 & & & \\
\hline
\end{tabular}

\section{Regresi Data Panel}

Chaw Test

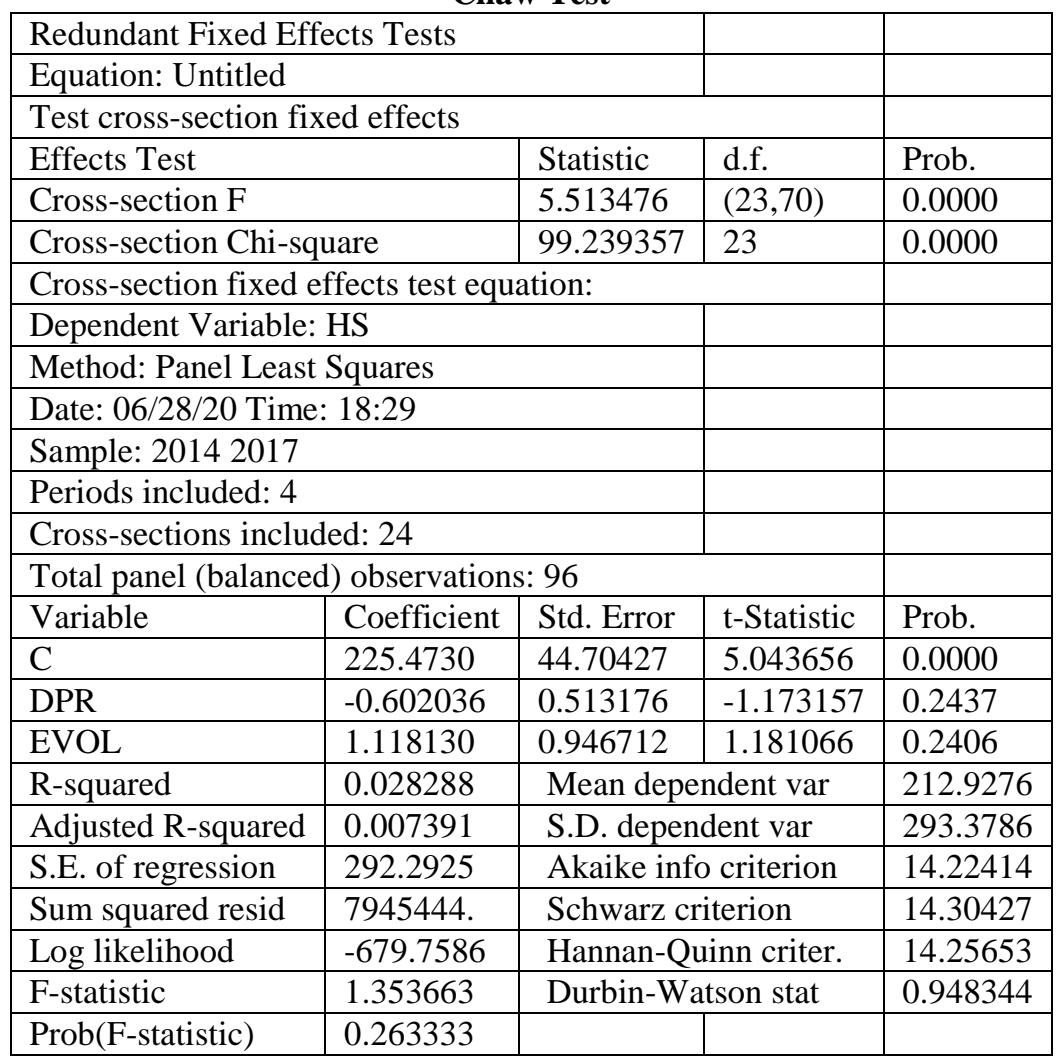


Hausman Test

\begin{tabular}{|c|c|c|c|c|}
\hline \multicolumn{4}{|c|}{ Correlated Random Effects - Hausman Test } & \\
\hline \multicolumn{4}{|c|}{ Equation: Untitled } & \\
\hline \multicolumn{4}{|c|}{ Test cross-section random effects } & \\
\hline \multicolumn{2}{|c|}{ Test Summary } & Chi-Sq. Statistic & Chi-Sq. d.f. & Prob. \\
\hline \multicolumn{2}{|l|}{ Cross-section random } & 6.243702 & 2 & 0.0441 \\
\hline \multicolumn{5}{|c|}{ Cross-section random effects test comparisons: } \\
\hline Variable & Fixed & Random & Var(Diff.) & Prob. \\
\hline DPR & 0.105867 & -0.103962 & 0.014973 & 0.0864 \\
\hline EVOL & 3.410956 & 2.686071 & 0.102574 & 0.0236 \\
\hline \multicolumn{4}{|c|}{ Cross-section random effects test equation: } & \\
\hline \multicolumn{3}{|c|}{ Dependent Variable: HS } & & \\
\hline \multicolumn{3}{|c|}{ Method: Panel Least Squares } & & \\
\hline \multicolumn{3}{|c|}{ Date: 06/28/20 Time: $18: 30$} & & \\
\hline \multicolumn{3}{|c|}{ Sample: 20142017} & & \\
\hline \multicolumn{5}{|c|}{ Periods included: 4} \\
\hline \multicolumn{5}{|c|}{ Cross-sections included: 24} \\
\hline \multicolumn{4}{|c|}{ Total panel (balanced) observations: 96} & \\
\hline Variable & Coefficient & Std. Error & t-Statistic & Prob. \\
\hline $\mathrm{C}$ & 143.0186 & 36.11663 & 3.959909 & 0.0002 \\
\hline DPR & 0.105867 & 0.424554 & 0.249360 & 0.8038 \\
\hline \multirow[t]{2}{*}{ EVOL } & 3.410956 & 0.869662 & 3.922165 & 0.0002 \\
\hline & Effects Spec & fication & & \\
\hline \multicolumn{4}{|c|}{ Cross-section fixed (dummy variables) } & \\
\hline R-squared & 0.654388 & \multicolumn{2}{|c|}{ Mean dependent var } & 212.9276 \\
\hline Adjusted R-squared & 0.530955 & \multicolumn{2}{|c|}{ S.D. dependent var } & 293.3786 \\
\hline S.E. of regression & 200.9257 & \multicolumn{2}{|c|}{ Akaike info criterion } & 13.66956 \\
\hline Sum squared resid & 2825980. & \multicolumn{2}{|c|}{ Schwarz criterion } & 14.36407 \\
\hline Log likelihood & -630.1389 & \multicolumn{2}{|c|}{ Hannan-Quinn criter. } & 13.95029 \\
\hline F-statistic & 5.301572 & \multicolumn{2}{|c|}{ Durbin-Watson stat } & 2.162827 \\
\hline Prob(F-statistic) & 0.000000 & & & \\
\hline
\end{tabular}

Fixed Efect Modle

\begin{tabular}{|c|c|c|c|c|}
\hline \multicolumn{5}{|c|}{ Dependent Variable: HS } \\
\hline \multicolumn{5}{|c|}{ Method: Panel Least Squares } \\
\hline \multicolumn{5}{|c|}{ Date: $06 / 28 / 20$ Time: $18: 28$} \\
\hline \multicolumn{5}{|c|}{ Sample: 20142017} \\
\hline \multicolumn{5}{|l|}{ Periods included: 4} \\
\hline \multicolumn{5}{|c|}{ Cross-sections included: 24} \\
\hline \multicolumn{5}{|c|}{ Total panel (balanced) observations: 96} \\
\hline Variable & Coefficient & Std. Error & t-Statistic & Prob. \\
\hline $\mathrm{C}$ & 143.0186 & 36.11663 & 3.959909 & 0.0002 \\
\hline DPR & 0.105867 & 0.424554 & 0.249360 & 0.8038 \\
\hline \multirow{2}{*}{ EVOL } & 3.410956 & 0.869662 & 3.922165 & 0.0002 \\
\hline & \multicolumn{3}{|c|}{ Effects Specification } & \\
\hline \multicolumn{5}{|c|}{ Cross-section fixed (dummy variables) } \\
\hline R-squared & 0.654388 & \multicolumn{2}{|c|}{ Mean dependent var } & 212.9276 \\
\hline Adjusted R-squared & 0.530955 & \multicolumn{2}{|c|}{ S.D. dependent var } & 293.3786 \\
\hline S.E. of regression & 200.9257 & \multicolumn{2}{|c|}{ Akaike info criterion } & 13.66956 \\
\hline Sum squared resid & 2825980. & \multicolumn{2}{|c|}{ Schwarz criterion } & 14.36407 \\
\hline Log likelihood & -630.1389 & \multicolumn{2}{|c|}{ Hannan-Quinn criter. } & 13.95029 \\
\hline F-statistic & 5.301572 & \multicolumn{2}{|c|}{ Durbin-Watson stat } & 2.162827 \\
\hline Prob (F-statistic) & 0.000000 & & & \\
\hline
\end{tabular}

\section{DISCUSSION}

Based on the data the researcher obtained and tested the data, processed the data and tested the model, the results showed that the best regression model used was the Fixed Effect Model. The following are the results of processing panel data with the Fixed Effect Model. 


\begin{tabular}{|c|c|c|c|c|}
\hline \multicolumn{5}{|c|}{$\begin{array}{l}\text { Dependent Variable: HS } \\
\text { Method: Panel Least Squares } \\
\text { Date: 06/28/20 Time: } 18: 28 \\
\text { Sample: } 20142017 \\
\text { Periods included: } 4 \\
\text { Cross-sections included: } 24 \\
\text { Total panel (balanced) observations: } 96\end{array}$} \\
\hline Variable & Coefficient & Std. Error & t-Statistic & Prob. \\
\hline $\mathrm{C}$ & 143.0186 & 36.11663 & 3.959909 & 0.0002 \\
\hline DPR & 0.105867 & 0.424554 & 0.249360 & 0.8038 \\
\hline \multirow[t]{2}{*}{ EVOL } & 3.410956 & 0.869662 & 3.922165 & 0.0002 \\
\hline & \multirow{2}{*}{\multicolumn{3}{|c|}{$\begin{array}{l}\text { Effects Specification } \\
\text { ummy variables) }\end{array}$}} & \\
\hline Cross-section fixed (dummy variables) & & & & \\
\hline R-squared & 0.654388 & \multirow{2}{*}{\multicolumn{2}{|c|}{$\begin{array}{l}\text { Mean dependent var } \\
\text { S.D. dependent var }\end{array}$}} & 212.9276 \\
\hline Adjusted R-squared & 0.530955 & & & 293.3786 \\
\hline S.E. of regression & 200.9257 & \multicolumn{2}{|c|}{ Akaike info criterion } & 13.66956 \\
\hline Sum squared resid & 2825980. & \multicolumn{2}{|c|}{ Schwarz criterion } & 14.36407 \\
\hline Log likelihood & -630.1389 & \multirow{2}{*}{\multicolumn{2}{|c|}{$\begin{array}{l}\text { Hannan-Quinn criter. } \\
\text { Durbin-Watson stat }\end{array}$}} & 13.95029 \\
\hline F-statistic & 5.301572 & & & 2.162827 \\
\hline Prob(F-statistic) & 0.000000 & & & \\
\hline
\end{tabular}

The best model used in this study is the Fixed Effect Model, with the form of the regression equation as follows:

$$
\mathrm{Y}=143,0186+0,105867 \mathrm{X} 1+3,410956 \mathrm{X} 2+\mathrm{e}
$$

Effect of Dividend Payout Ratio on Stock Prices on industrial companies listed on the Indonesia Stock Exchange 2014-2017. The results of data processing obtained a regression coefficient of $0.105867 \mathrm{t}$ test value is 0.249360 and the probability value is 0.8038 . The coefficient value of 0.105867 means that it has a positive effect, the value of $t$ count $0.249360<\mathrm{t}$ table 2.07387 and the probability value of $0.8038>0.05$ means that the Dividend Payout Ratio has no significant effect on the Stock Price.

The Influence of Earning Volatility on Stock Prices in industrial companies listed on the Indonesia Stock Exchange 2014-2017. The results of Earning Volatility processing has a regression coefficient of 3.410956, the t-test value is 3.922165 and the probability value is 0.0002 The coefficient value of 3.410956 means that it has a positive effect, the value of $\mathrm{t}$ arithmetic $3.922165>\mathrm{t}$ table 2.07387 and a probability value of $0.0002<0.05$ means that it has a significant effect, So it can be concluded that Earning Volatility partially has a significant positive effect on the Stock Price

The Effect of Dividend Payout Ratio and Earning Volatility on Share Prices in industrial companies listed on the Indonesia Stock Exchange $2014-2017$ is $65.4 \%$. Obtained $F$ value of 5.301572 with a level of $\alpha=5 \%$, df numerator $3-1=2$ and $\mathrm{df}$ denominator 96-2-1 $=93$ then the $\mathrm{F}$ value of the table is 3.09 so that the F count 5.301572> F table 3.09 and the value of probability of $0.000000<0.05$ which means that Dividend Payout Ratio and Earning Volatility together have a significant effect on stock prices.

\section{CONCLUSION}

Dividend Payout Ratio partially has no significant effect on stock prices. However Earning Volatility partially has a significant positive effect on Stock Prices. Then the Dividend Payout Ratio and Earning Volatility together have a significant effect on stock prices. As such, investors in deciding to invest in technical analysis are more dominant than technical analysis.

\section{REFERENCE}

1. Rusdin. (2008). Pasar Modal: Teory, Masalah Dan Kebijakan dalam Praktik. Bandung: Alfabeta.

2. Bambang, R. (2011). Dasar Dasar Pembelanjaan Perushaan. Edisi Keempat. Yogyakarta: BPFE.

3. Hartono, J. (2005). Analisis dan Desain Sistem Informasi: pendekatan terstruktur teori dan praktek aplikasi bisnis. Yogyakarta: Andi.

4. Brigham, E. F., \& Joel, F. H. (2009). Fundamentals of Financial Management, 12th edition. South-Western Cengage Learning 5191 Natorp Boulevard Mason, OH 45040 USA.

5. Sartono, A. (2008). Understanding Indonesia: Does it really matter.

6. Sutrisno, E. (2009). Manajemen Sumber Daya Manusia Edisi pertama. Jakarta: Kencana Prenada Media Group.

7. Darmadji, T., \& Fakhruddin, H. M. (2006). Pasar Modal Indonesia. Jakarta: Salemba Empat.

8. Van Horne, J. C., \& Wachowicz, J. M. (2008). Fundamentals of Financial Management. Thirteenth edition: published under by Prentice Hall, Inc.

9. Ningtyas, C. P., Suhadak, S., \& Nuzula, N. F. (2016). Evaluasi kinerja dan perbandingan analisis rasio (Studi pada Sektor Makanan dan Minuman yang Terdaftar di Bursa Efek Indonesia Periode 2010-2014). Jurnal Bisnis dan Manajemen, 3(2). 
10. https://corporatefinanceinstitute.com

11. Suwanda, H. (2013). Rahasia Bebas Finansial Dengan Berinvestasi di Pasar Modal. Jakarta: PT. Gramedia Pustaka Utama.

12. Khuriaji, A. W., \& dan Surya, R. (2013). Hubungan Kebijakan Dividen (Dividend Payout Ratio dan Dividend Yield) terhadap Volatilitas Harga Saham di Perusahaanperushaaan yang Terdaftar di Bursa Efek Indonesia. Diponegoro Journal of Accounting. 2(3).

13. Theresia, P., \& Arilyn, E. J. (2015). Pengaruh dividen, ukuran perusahaan, hutang jangka panjang, earning volatility, pertumbuhan aset, trading volume, dan kepemilikan manajerial terhadap volatilitas harga saham. Jurnal Bisnis dan Akuntansi, 17(2), 197-204.

14. Subiyakti, A. N. (2017). Pengaruh earnings volatility terhadap earnings response coefficient pada saat konvergensi ifrs di indonesia. E-Journal Akuntansi" EQUITY", 3(3).

15. Lubis, A. S. (2019). Pengaruh Earning Volatility dan Leverage terhadap Volatilitas Harga Saham Sektor Property dan Real Estate yang terdaftar di Bursa Efek Indonesia. JOM FISIP Vol 6: Edisi II Juli-Desember 2019, 1-15.

16. Takarini, N., \& Hamidah, H. (2011). Rasio Keuangan Dan Pengaruhnya Terhadap Harga
Saham Perusahaan Yang Terdaftar Dijakarta Islamic Index. Journal of Bussiness and Banking. 1(2):93-104.

17. Darmadji, T., \& Fakhruddin, H. M. (2011). Pasar modal di indonesia. Jakarta: Salemba Empat.

18. Hadi, N. (2013). Pasar Modal (Acuan Teoritis dan Praktis Investasi di Instrumen Keuangan Pasar Modal). Yogyakarta: Graha Ilmu.

19. Zulfikar. (2016). Pengantar Pasar Modal dengan Pendekatan Statistika. Yogyakarta: Deepublish.

20. Widoatmodjo, S. (2011). Cara Sehat Investasi di Pasar Modal. Jakarta: Pt Elex Media.

21. Jogiyanto. (2014). Teori Porfolio dan Analisis Investasi, Yogyakarta: BPFE-UGM.

22. Sugiyono, F. X. (2017). Instrumen Pengendalian Moneter: Operasi Pasar Terbuka (Vol. 10). Pusat Pendidikan Dan Studi Kebanksentralan (PPSK) Bank Indonesia.

23. Sugiyono. (2018). Metode Penelitian Kuantitatif, Kualitatif, dan R\&D. Bandung: Alfabeta.

24. Sugiyono, A. (2016). Outlook Energy Indonesia 2016-Energy Development in Supporting Green Industri. PTSEIK-BPPT, Jul-2016.

25. Basuki, A. T., \& dan Nano, P. (2016). Analisis Regresi Dalam Penelitian Ekonomi \& Bisnis, Edisi Pertama. Jakarta: Rajawali Pers. 